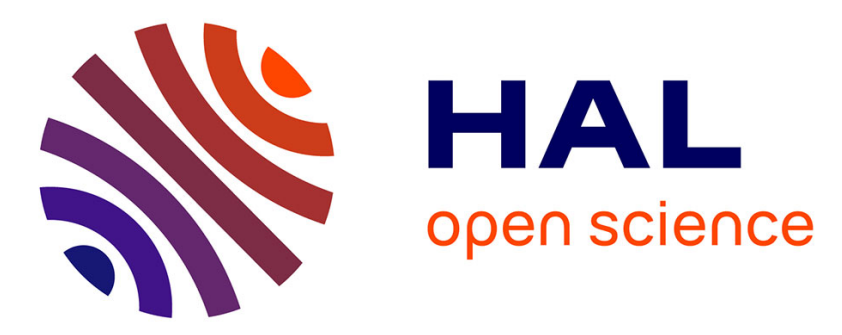

\title{
Ultrasound image-based comanipulation for enhanced perception of the contacts with a distal soft organ
}

Cecile Torterotot-Poquet, Marie-Aude Vitrani, Pierre Mozer, Guillaume Morel

\section{To cite this version:}

Cecile Torterotot-Poquet, Marie-Aude Vitrani, Pierre Mozer, Guillaume Morel. Ultrasound imagebased comanipulation for enhanced perception of the contacts with a distal soft organ. 2011, 10.1109/ROBIO.2011.6181441 . hal-01170729

\section{HAL Id: hal-01170729 \\ https://hal.sorbonne-universite.fr/hal-01170729}

Submitted on 2 Jul 2015

HAL is a multi-disciplinary open access archive for the deposit and dissemination of scientific research documents, whether they are published or not. The documents may come from teaching and research institutions in France or abroad, or from public or private research centers.
L'archive ouverte pluridisciplinaire HAL, est destinée au dépôt et à la diffusion de documents scientifiques de niveau recherche, publiés ou non, émanant des établissements d'enseignement et de recherche français ou étrangers, des laboratoires publics ou privés. 


\title{
Ultrasound image-based comanipulation for enhanced perception of the contacts with a distal soft organ
}

\author{
Cecile Torterotot, Marie-Aude Vitrani, Pierre Mozer and Guillaume Morel
}

\begin{abstract}
In this paper, the concept of ultrasound imagebased force enhancement is introduced. It consists of a robotic comanipulation system that assists a surgeon performing an ultrasound exam through a minimally invasive access. The robot is programmed in such a way that the surgeon's feeling of the distal contact between the probe and the organ is enhanced, despite disturbances raising from interactions with the trocar or the natural orifice through which the probe is inserted.

Contrarily to existing approaches for force enhancement, the proposed method does not require any distal, nor proximal force sensor. Rather, forces to be applied by the comanipulator are computed from the measurement of the organ deformations in the ultrasound image. In the paper, an example application to computer-assisted prostate biopsy procedures is given. A first implementation of the concept is then presented, involving an endorectal ultrasound probe and a one degree of freedom robot. In vitro experiments validate that the proposed mode of assistance significantly helps the detection of contacts between the probe and a balloon simulating a prostate despite large friction disturbances.
\end{abstract}

\section{INTRODUCTION}

Comanipulation is a paradigm for robotic assistance to gesture in which both a human operator and a robot simultaneously contribute to the execution of the same task by acting on the same tool. This approach is of particular interest in the context of medical applications, where the robot can help enhancing the surgeon manipulation capabilities. Comanipulators can bring a number of assistance functions, ranging from guidance by virtual fixtures (see e.g. [1] in the domain of orthopedic surgery) to tremor filtering (see e.g. [2] or [3] in the domain of eye surgery) or dexterity enhancement (see e.g. [4] in the domain of laparoscopic surgery). In this paper, we focus on a particular function of comanipulation called force scaling.

Force scaling is a function in which the robot is used to provide the operator with a scaled feedback of the force applied to the environment. When the scaling factor $\lambda$ is smaller than one, the robot operates force amplification, in that sense that the force applied to the environment is amplified as compared to the force applied by the operator. Force amplification has been introduced early in the 1990's, in the context of power exoskeletons [5], and industrial robotics [6], where the robot had to help a worker in carrying large payloads. Similar control approaches are used in these two pioneer papers. They are based on the equation that

All authors are with Institut des Systèmes Intelligents et de Robotique, Univ. Pierre \& Marie Curie - Paris 6, CNRS UMR 7222, Paris, France firstname.lastnamedisir.upme.fr

Pierre Mozer is also with La Pitié-Salpêtrière hospital, Urology Dpt., Paris, France characterizes the equilibrium of a comanipulated tool:

$$
f_{r}+f_{u}+f_{e}=0,
$$

where $f_{r}, f_{u}$ and $f_{e}$ are the forces applied by the robot, the user and the environment to the tool, respectively. By measuring two of these three forces, it is always possible to control the robot in such a way that, at the equilibrium, $f_{u}=-\lambda f_{e}$. For example, in [5], two sensors measure independently $f_{u}$ and $f_{e}$ and the mixed signal $f_{u}+\lambda f_{e}$ is servoed to zero thanks to the action of the robot. Note that beyond the equilibrium condition (1) that supposes a stable behavior, force amplification induces stability problems that are specific to the tripartite interaction dynamics, which were more recently explored in [7].

Force scaling can also provide force enhancement when selecting $\lambda>1$. This is useful when very small forces have to be applied to the environment, leading, for the user, to a poor perception of the interaction. For example, in [8], a system called Steady Hand is presented for force enhancement in the context of eye micro-surgery. To this aim, a micro force sensor is developed to measure forces applied to the eye, while a commercially available sensor equips the user interface handle. The controller is based on an inner velocity loop and an outer force loop mixing, again, the two force signals.

An interesting extension of the force enhancement principle can be implemented for applications in minimally invasive surgery. Here, the instrument is inserted through either a natural orifice or a trocar, whereas its tip interacts with organs inside the patient. In this case, the environment force $f_{e}$ is composed of two forces: a force $f_{o}$ produced by the interaction with the organ and a disturbance force $f_{d}$ produced by the physical interaction with either the trocar or the natural orifice and surrounding tissues along instrument body. Therefore Eq. (1) rewrites:

$$
f_{r}+f_{u}+f_{o}+f_{d}=0,
$$

During unassisted surgery, $f_{r}=0$ and the surgeon feels the sum of the force applied to the organ and the disturbing force. The disturbing forces can be rather large and significantly alter the perception of the organ force [9]. Here, the force enhancement scheme appears to be of particular interest. Indeed, assuming that both $f_{u}$ and $f_{o}$ are measured, then $f_{u}+\lambda f_{o}$ can again be servoed to zero. In this case, a simple high static gain controller (e.g. PI control) allows not only to scale the force applied by the user but also to reject the disturbance force $f_{d}$. This principle was implemented in [10] in the context of laparoscopic surgery, where a particular 
sensory device, described in [11], was used to measure $f_{o}$ from a sensor mounted on the trocar.

Unfortunately, in most applications of minimally invasive surgery, which include endoluminal interventions, measuring $f_{o}$ is not possible because no force sensor can be added at the tool tip due to miniaturization, sterilization and economical constraints. To cope with this problem, the idea was launched a few years ago to estimate forces from real time imaging, which is always available in the context of minimally invasive surgery. It was implemented in the context of force feedback teleoperation in [12], where marks are made on a deformable membrane which boundaries are fixed on a frame. A camera which is fixed with respect to the frame and an image processing software provide the points displacements. These are the entries of a finite elements model, which returns the interaction force between the tool tip and the membrane at the contact point (from which the robot command is computed). An other way to proceed in to use snakes to extract the body shape from its image as in [13], which also uses a camera and a deformable body that are fixed in the reference space. Organ deformations can also be estimated from 3D ultrasound imaging, as in [14], but the time needed for acquiring and processing 3D ultrasound images seems incompatible with real time force feedback. In the present paper, the principle of ultrasound imagebased force enhancement by comanipulation is introduced. It consists of mixing the force enhancement approach and the ultrasound image-based force estimation. To the authors' knowledge, this is the first time that image-based force enhancement by comanipulation is reported in the literature. Additional originality arise from the fact that: i) the comanipulated tool is the imaging device, namely an ultrasound probe; ii) a fairly backdrivable robot is used, which allows for controlling the system without any force sensor. The general concept of ultrasound image-based force enhancement is first presented in Sec. II. An example application of this research, which is the assistance to prostate biopsy is then presented in Sec. III. A 1 Degree of Freedom (DOF) apparatus is presented in Sec. IV, on which experiments were conducted. Experimental results are presented in Sec. V. These experiments emphasize a property of comanipulation force enhancement: since the sensed forces are amplified while the user and organ movements are at the same scale, the apparent stiffness of the contacted organ is amplified. Experiments are conducted with naive subjects in order to evaluate if the sensed stiffness is properly affected by the system.

\section{ULTRASOUND IMAGE-BASED FORCE ENHANCEMENT}

\section{A. Overview of the proposed approach}

The problem being studied is described in Fig. 1: a 2D end-fire planar ultrasound probe is being manipulated by both a surgeon and a robot. It is inserted in a patient through a minimally invasive access that can be a trocar or a natural orifice. The distal ultrasound transducer is contacting an organ for imaging purposes. In such a configuration, it may happen that the perception of the probe-organ contact force is not easily felt by the surgeon due to disturbance forces applied on the insertion device through the minimally invasive access. This may affect the quality of the examination gesture, leading to excessive deformation of the organ and difficulty in stabilizing the probe under good observation conditions. The goal is then, for the robot, to enhance the force sensed by the surgeon.

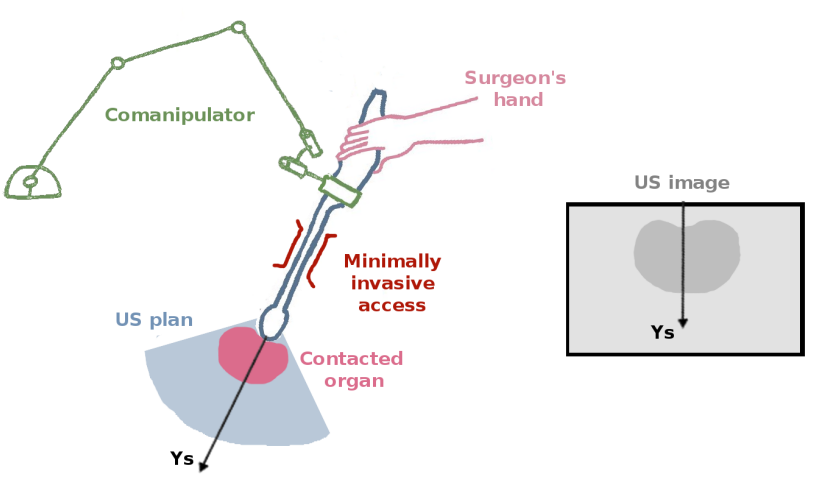

Fig. 1. A comanipulated ultrasound probe is inserted into patient's body and presses an organ

A frame $\mathcal{F}_{s}=\left(O_{s},\left(\vec{x}_{s}, \vec{y}_{s}, \vec{z}_{s}\right)\right)$ is attached to the probe, $\left(O_{s}, \vec{y}_{s}\right)$ corresponding to the vertical direction of the ultrasound image, along which the pressure has to be controlled for proper imaging. Assuming that, for proper organ examination, $\left(O_{s}, \vec{y}_{s}\right)$ corresponds to the normal of the organ, then the force control problem can be considered to be one-dimensional along $\left(O_{s}, \vec{y}_{s}\right)$. Along this direction, the dynamics can be written as:

$$
f_{u}+f_{r}+f_{p}=f_{u}+f_{r}+f_{o}+f_{d}=M \ddot{y}
$$

where $M$ is the device mass. At the equilibrium, when $\ddot{y}=0$, Eq. (2) holds.

\section{B. Force enhancement without force sensor}

The system is not equipped with any force sensor and therefore the conventional force enhancement control schemes cannot be implemented. Rather:

- an estimation of the deformation $d$ of the organ is extracted from the ultrasound image in order to substitute for the absence of measurement for $f_{o}$, see Sec. II-C.

- The force $f_{r}$ exerted by the robot is controlled in open loop by imposing an equivalent force to the actuator torques $\tau_{a}$.

Namely, considering that the robot dynamics write:

$$
-J^{T}(q) f_{r}+\tau_{a}=H(q) \ddot{q}+B(q, \dot{q}) \dot{q}+\tau_{f}+\tau_{g}(q),
$$

where $q$ is the robot joint position vector, $J(q)$ is the so-called jacobian matrix of the robot mapping the joint velocities into the end effector velocities, $[H(q) \ddot{q}+B(q, \dot{q}) \dot{q}]$ groups the inertial, Coriolis and centrifugal torques, $\tau_{f}$ and $\tau_{g}(q)$ are the joint friction torque and the gravity torque, respectively, it is possible to define a new control input $f_{a}$, which is the force to be applied by the robot, and to compute:

$$
\tau_{a}=J^{T}(q) f_{a}+\hat{\tau}_{g}(q)
$$


where $\hat{\tau}_{g}(q)$ is an estimate of the gravity torque. As a result, under static conditions:

$$
f_{r}=f_{a}+f_{r d}
$$

where $f_{r d}=J^{-T}(q)\left[\hat{\tau}_{g}(q)-\tau_{q}(q)-\tau_{f}\right]$ represents the residual amount of friction and uncompensated weight in the robot structure. This open loop approach is conventionally used for haptic devices and provides satisfactory force control as long as a robot exhibiting low inertia and low joint friction is used.

\section{Estimation of the deformation $d$ from ultrasound images}

In this paper, a real time 2D imaging device is considered, which produces an image of a planar slice of the observed organ. Referring to Fig. 2, estimating the deformation consists of extracting the distance $d$, measured along axis $\left(O_{s}, \vec{y}_{s}\right)$, between the undeformed organ surface and the organ surface in the current image. This corresponds to how much the probe "penetrates" in the virtually undeformed organ.

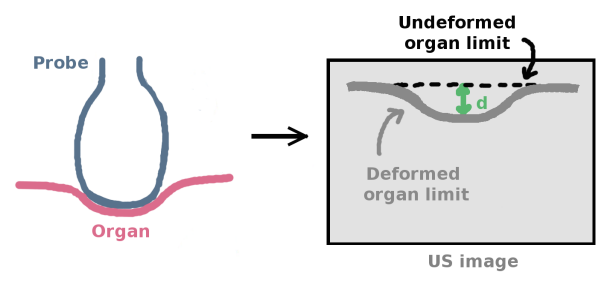

Fig. 2. Vizualiation of the definition of $d$

Estimating this displacement has been solved in the literature considering the example of prostate 3D ultrasound imaging. In [14], a robust registration algorithm is used to register two $3 \mathrm{D}$ ultrasound images of a prostate in the presence of deformations due to the pressure exerted by the endorectal probe on the prostate through the rectum wall. Unfortunately, this algorithm can hardly be exploited in real time and would require $3 \mathrm{D}$ ultrasound real time imaging. Nevertheless, these results demonstrate the feasibility of estimating $d$ from ultrasound images in the clinical context, since the technology presented in [14] is today available for clinical use thanks to the Urostation system commercialized by Koelis.

Generally speaking, developing a robust algorithm for estimating $d$ from 2D images strongly depends on the type of available images, which in turns depends on the properties of the observed organ. For example, image quality strongly differs between endorectal prostate imaging and laparoscopic ultrasound imaging of the liver. The development of a robust image processing algorithm for in vivo real time extraction of $d$ is thus specific to the targeted clinical application and is in fact beyond the scope of the present paper, which focuses on the general concept of image-based force enhancement. Nevertheless, for illustration purpose, an example algorithm will be described in Sec. IV when presenting the system developed for experimental validations, where the problem is simplified by the fact that high contrast images of the entire phantom soft organ are obtained for these in vitro experiments.
In the rest of the paper, it will be assumed that $d$ can be extracted from images. A major problem lies in the low frequency at which the information $d$ is made available from image grabbing and processing. Meanwhile, the deformation $d$ must be available for the controller at a high rate, because the human skin is able to discriminate two tactile events separated by $10 \mathrm{~ms}$ [15]. To cope with this problem, between two measurements of $d$ an estimation $\hat{d}$ can computed by:

$$
\hat{d}(t)=d\left(t_{0}\right)+\delta y(t)
$$

where $t_{0}$ is the time of the last measurement of $d$ and $\delta y(t)$ is the displacement of $O_{s}$ along $\vec{y}_{s}$ between $t_{0}$ and $t$, which can be obtained from the robot encoders.

\section{Control law}

The control law is aimed at computing a force $f_{r}$ that the robot shall exert in order to help the surgeon feeling the force applied on the organ. It is presented in Fig. 3. An extremely simple controller is used:

$$
f_{a}=-k \hat{d}
$$

where $k$ is a positive gain that physically corresponds to a stiffness. It is important to notice that $k$ does not correspond to any model of the organ. Rather, it can be viewed as a virtual stiffness, added in parallel with the organ in such a way that it increases the apparent stiffness of the organ. With this controller, the equilibrium condition becomes:

$$
f_{u}=k \hat{d}-f_{r d}-f_{d}-f_{o} .
$$

Note that contrarily to the force enhancement method presented in [11], neither the disturbance force $f_{d}$ coming from the interaction at the minimally invasive access, nor the disturbance force $f_{r d}$ arising from friction and uncompensated gravity in the robot, are canceled by the controller. This is due to the fact that no force sensor is used. If a disturbance cancellation was desired, a possibility would be to equip the user interaction port with a force sensor measuring $f_{u}$ and to servo the mixed signal $\left(f_{u}-k \hat{d}\right)$ to zero. However, this would add cost and complexity to the system for a benefit that is not a priori obvious.

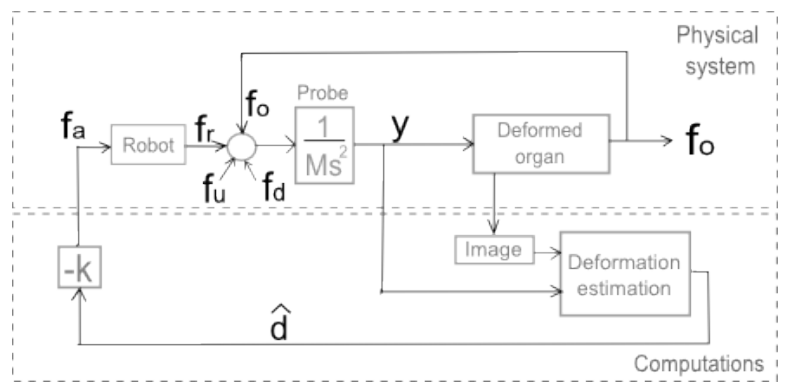

Fig. 3. Control scheme

Indeed, if the stiffness $k$ is large enough, the forces generated by a given deformation of the organ will be sensed by the surgeon, even for small deformations, or in the presence of large friction. An important point here is that the function to 


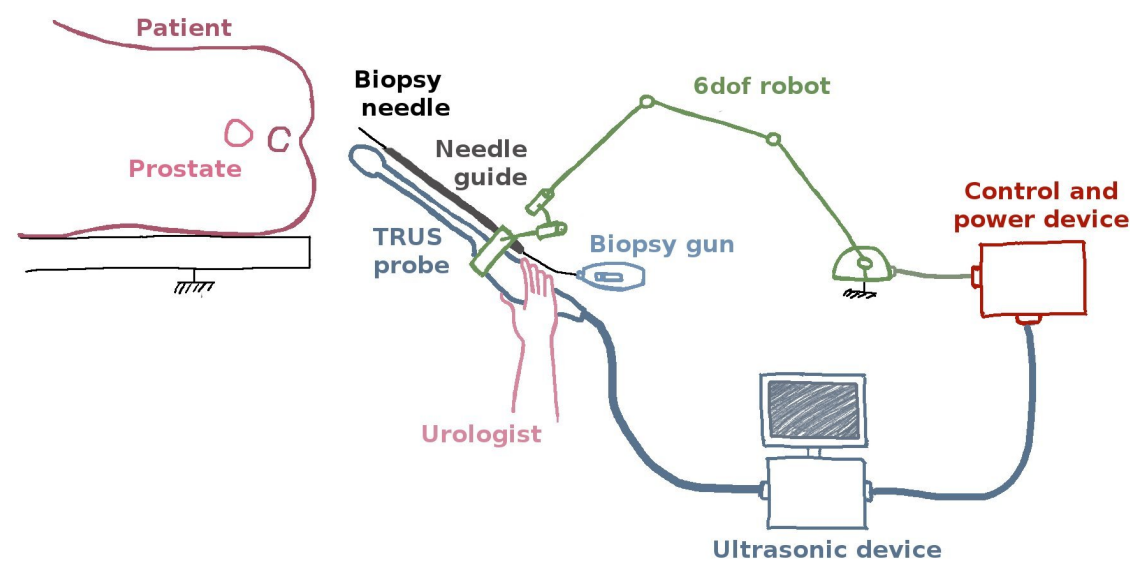

Fig. 4. Ultrasound image based comanipulated system proposed as an assistance to prostate biopsy

be realized by the robot is not to restore high fidelity sensing of the contact force, but rather to enhance the perception of contacts. For this reason, there is no need for the behavior implemented thanks to Eq. (9) to be realistic from the biomechanical point of view.

Assuming that, for small deformations, the organ can be modeled by pure stiffness $k_{o}$ :

$$
f_{o}(d)=-k_{o} d,
$$

then the apparent stiffness for the user, defined as the ratio between a force and a displacement, will be:

$$
k_{a}=k_{0}+k \text {. }
$$

\section{DESCRIPTION OF AN EXAMPLE APPLICATION}

A possible application field for the apparent stiffness modification is the prostate biopsy process. The prostate is a gland that is part of the masculine reproductive system and in which cancerous cells can develop. The medical examination which allows diagnosing a prostate cancer is prostate biopsy. In 2010, 217,730 new prostate cancer cases have been detected and more than one million biopsies have been performed in the USA [16]. Considering this pathology extend, any improvement in the prostate biopsy process could have important effects on patient's life expectancy and quality of life.

Each urologist has her/his own way to proceed, but general guidelines are given [17]. During a prostate biopsy, the patient preferably lies on his flank. An endorectal ultrasonic probe fitted with a needle guide is inserted in the patient rectum. A local anesthesia is then preformed. Using the ultrasound 2D image, the surgeon points the needle in the desired direction, pierces by hand the rectum surface and activates the biopsy gun. Biopsies are distributed in the prostate following a sextant scheme.

The good distribution of biopsies in the prostate is the key aspect of the procedure as it limits the risk not to detect an existing tumor and the risk to overestimate a tumor extend. During standard examination, the surgeon has to construct a mental 3D volume of the prostate using the $2 \mathrm{D}$ cross section provided by the ultrasound image. This is rather challenging, due to the mobility and deformations experienced by the prostate when moving the probe or inserting the needle, or caused by the patient motions [18].

This is why navigation assistants are being developed and commercialized, like the ultrasound-based registration system proposed in [14] and commercialized by Koelis. This system allows to locate the probe with respect to a reference (initial) prostate frame, and displays to the urologist the distribution of the biopsies on a 3D prostate image. The algorithms used for 3D registration are robust to prostate deformations up to a certain amount. However, if a large force is applied to the prostate, the $3 \mathrm{D}$ registration precision can be affected. In practice, due to the friction $f_{d}$ occurring between the rectum and the probe, the urologist does not accurately feel the force $f_{o}$ that he/she is applying on the prostate. In this context, providing assistance to the urologist in order to increase the sensation of the forces applied to the prostate helps limiting the deformation and thus eases the registration.

In order to implement the ultrasound image-based force enhancement function, a robotic system is being developed for assistance to prostate biopsy, as depicted in Fig. 4. It uses a conventional ultrasound probe fitted with a needle guide. The probe is manipulated jointly by the urologist and a robotic arm. This robotic arm has $6 \mathrm{DOF}$, among which 3 are motorized and allow the positioning of one point $P$ of the robot wrist, coinciding with one point of the probe. The other 3 DOF constitute a free revolute joint and are equipped with position sensors. The kinematics of the robot respects the kinematic constraints imposed by patient's anus. The robot control input is a force $f_{a}$ applied at point $P$, from which a static actuator torque $\tau_{a}$ is computed according to Eq. (5). 


\section{DEVELOPMENT OF A 1 DOF MOCKUP}

In order to evaluate the feasibility of ultrasound imagebased force enhancement in the context of assistance to prostate biopsy, a 1 DOF prototype was fabricated to comanipulate an endorectal ultrasound probe.

\section{A. Hardware}

The 1DOF comanipulation robot is presented in Fig. 5. This robot provides motion to the ultrasonic probe along its main axis within a range of approx. $10 \mathrm{~cm}$. A balloon filed with water and placed at a water filled tub bottom simulates the prostate. The tip of the probe must be immersed in water in order to provide a good quality ultrasonic image. The robot design ensures waterproofness thanks to a fixed structure holding the actuator above the tub and a transmission exploiting a plastic belt immersed in the water. The actuator is a Parvex RX120L permanent magnet motor, which offers a $0.285 \mathrm{Nm}$ maximum torque. A pulley and belt system transforms the rotative motion into a linear one and enables the robot to provide up to $29 \mathrm{~N}$ on the probe. The probe is fixed on a chariot guided by two steel bars. The motor is equipped with an encoder providing a measure of the probe position with a resolution lower than a hundredth of a millimeter.

It shall be noticed that this rapidly prototyped device exhibits a lot of friction (approx. $5 \mathrm{~N}$ ). For this reason, it was not necessary to implement a device simulating the friction of a minimally invasive access into the mockup. Indeed, as emphasized by Eq. (9), the robot friction and the rectum friction are equivalent disturbances to the system.
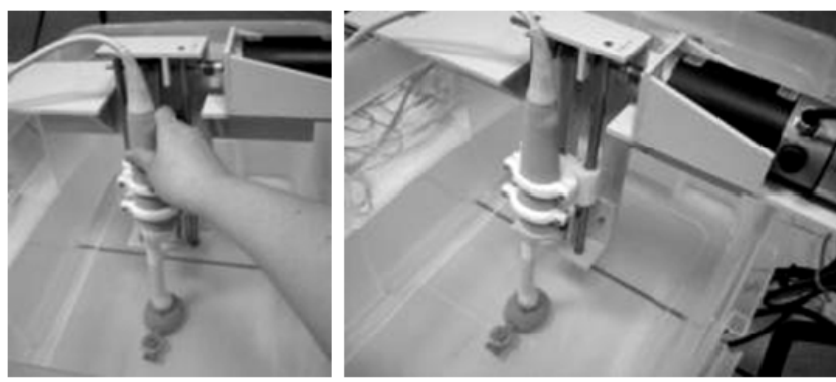

Fig. 5. Experimental apparatus

In order to implement the control algorithm and image processing, a dedicated controller hardware was developed, see Fig. 6. The image is obtained by an ultrasound scanner from Ultrasonix (Sonix SP, research version) and is then grabbed via the video-to-USB2 converter of ImagingSource, which is plugged in the Svideo B\&W output of the ultrasound scanner and provides a video flow by USB.

A first computer (Computer 1 in Fig. 6) is used to run the image processing at image grabbing rate (Typ. $25 \mathrm{~Hz}$ ) and sends the image-estimated balloon deformation, through an Ethernet link, to a second computer (Computer 2 in Fig. 6), running the control law described by Eq. (5), (7) and (8) at $1 \mathrm{kHz}$. Computer 2 includes the necessary ports to dialog with the robot motor boards and runs in real time under Linux RTAI (Real Time Application Interface).

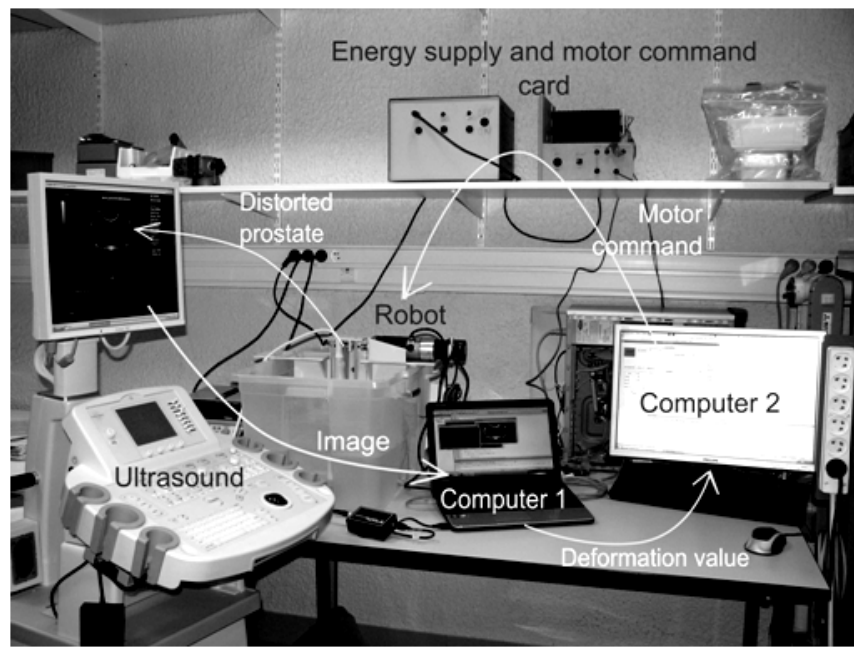

Fig. 6. Architecture for imaging and real time control

\section{B. Image processing for the estimation of $d$}

Although the development of robust algorithms for estimating the prostate deformation is beyond the scope of the present paper, if was necessary, for the purpose of experimental validation, to implement an example algorithm. In the proposed setup, ultrasound images of the balloon exhibit a very sharp contrast that eases contour detection.

The grabbed image is firstly cropped, in order to reduce the computational time. Then a threshold is used to produce an image where the balloon contour appears in white while the background appears in black. Finally, conventional morphological operators allow for improving the image quality. Thanks to an appropriate empiric choice of the threshold and morphological operators applied, this very simple method allows to eliminate ultrasound image artefacts while providing a contour with a limited number of discontinuities, see Fig.7. From the obtained image, deformation $d$ is to be computed. In fact, only the height $h$ of the balloon in the image is to be measured. The prostate deformation $d$ is further defined by:

$$
d=\frac{h_{0}-h}{h_{0}},
$$

where $h$ is the current prostate height along $\left(O_{c}, \vec{y}_{c}\right)$ and $h_{0}$ is the prostate height when no force is applied, that can be recorded prior to any contact experiment. In order to extract the balloon height from the image, a point is first placed in the middle of the image; exploring the pixels along $\left(O_{c}, \vec{y}_{c}\right)$ above and under the starting point until reaching a white pixel will produces a segment that corresponds to the balloon height, see Fig. 7.

Note that, for the experiments presented in Sec. V, the behavior of several subjects contacting a balloon under the assistance of a force enhancement device is compared. Since the balloon had to be periodically changed, its size was varying from an experiment to another. In order to provide a 
comparable behavior in spite of changes in the balloon size, it was decided to define $d$ as the relative deformation thanks to Eq. (12) rather than the absolute deformation previously defined in Fig. 2. This does not affect the rest of the control law providing that gain $k$ is appropriately scaled.
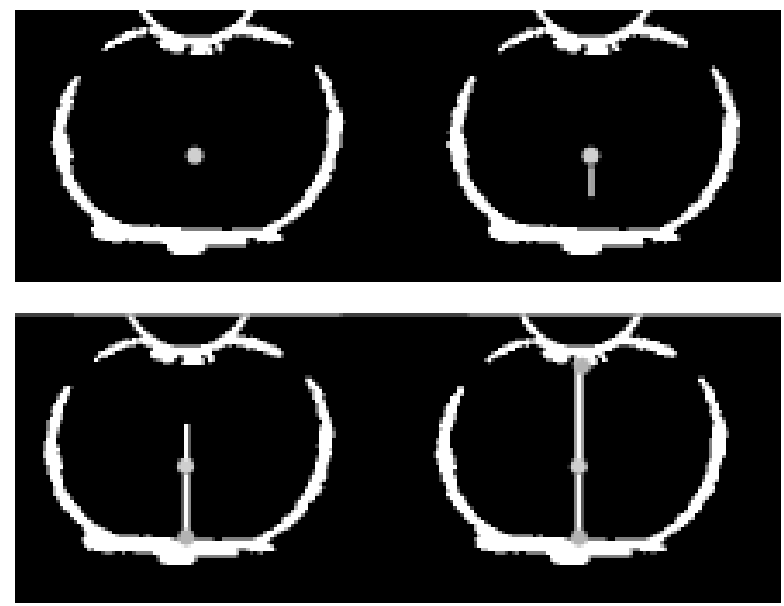

Fig. 7. Evolution of the algorithm computing the balloon height

\section{EXPERIMENTS}

\section{A. Protocol}

Experiments have been carried out with five naive subjects. In order to have them focused on what they felt with their sense of touch, they have been asked to close their eyes during the experiments.

Each subjects manipulated the robot under five different conditions:

- A: $f_{a}=0$.

- B: control law (8), with $k=k_{0}$, manually tuned to have a sensible effort on the user hand. Furthermore, during this experiment, the deformation estimation is not interpolated using the robot position between two image measurements.

- C: control law (8), with $k=2 k_{0}$. During this experiment, the deformation estimation is not interpolated using the robot position between two image measurements.

- D: Same as B $\left(k=k_{0}\right)$, but using encoder based interpolation between two image based measurements.

- E: Same as C $\left(k=2 k_{0}\right)$, but using encoder based interpolation between two image based measurements.

The subjects had to realize four trials for each control law. One trial consists in two distinct tasks:

- Touch task: the subject must manipulate the probe and stop his/her hand as soon as he/she thinks that the probe tip touches the balloon.

- Press task: the subject must press the balloon as strong as he/she wants, but is asked to reproduce the same force at each trial. Each subject thus chooses his/her own force; the five deformations obtained by a subject for a given law appeared to be in a small range, which shows the stability of the force exerted.

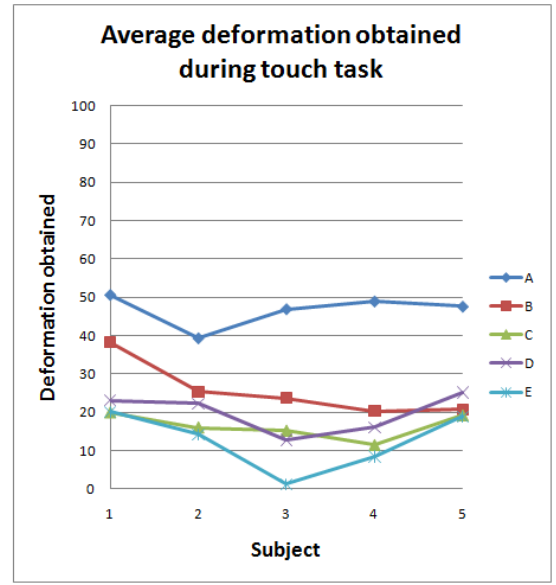

Fig. 8. Average balloon deformation obtained for each subject and each command law for touch task

It appeared that balloons suffer a lot from supporting important loads and being in the water that is their mechanical properties change and they become flabby. The balloon has so been changed between each subject.

\section{B. Results}

Results are presented in Fig. 8 and Fig. 9. It displays the average balloon deformation obtained by each subject for each law, for both tasks.

First, it appears that whatever the considered law, the tactile sensations are improved by the cobot use and, as a consequence, the gesture is improved: the balloon is less deformed for identical haptic sensations. In the case of touch task, the balloon is 15 to $43 \%$ less deformed than without comanipulation (law A). In the case of press task, the decrease is of 30 to $52 \%$.

It can also be seen that doubling the proportional coefficient used in the command law induces a reduction of 23 to $46 \%$ of the balloon deformation during both touch and press tasks. Interestingly, the results do not exhibit a marked benefit for the interpolation thanks to the encoder measurement (see Eq. 7), although the image acquisition frequency was rather low (less than $10 \mathrm{~Hz}$ ). A possible explanation is that the large friction experienced by the apparatus stabilizes the system despite low sampling frequency. In a real application, the friction between the probe and the patient's rectum is expected to bring the same stabilizing effect, increasing the safety of the approach.

\section{Discussion}

During the experiments, the subjects had to keep their eyes closed. They did not have any other source of information to estimate the balloon deformation than their sense of touch. For these reasons, the subjects were not disturbed by the delay between the image display and the force display, induced by image processing. For a manipulation using multimodality (sense of touch and sense of sight), we noticed that this delay can become disturbing. In the future, multimodal experiments will be conducted. Meanwhile, effort will 


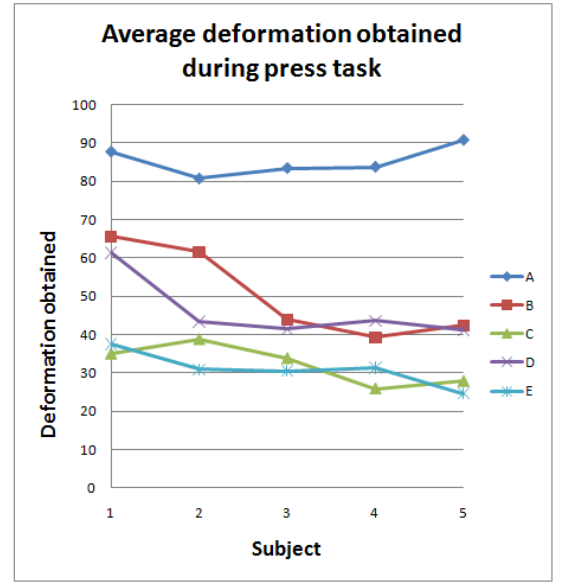

Fig. 9. Average balloon deformation obtained for each subject and each command law for press task

be put in reducing the delay by implementing all the image processing and the control law in one single computer.

When no interpolation is used for estimating the balloon deformation, a transient phase can be felt, induced by the low rate refreshments of $d$. For this reason, the second command type (using the motor encoder for interpolation) was reported by all the subjects to provide a more comfortable feedback. However, it did not affect the efficiency of the approach in enhancing the perception of organ-probe contact.

The transient phase could be smoothed, even perhaps to an imperceptible level, by adding a filter in the loop. The problem is that this could induce more delay and could make the system become unstable. We will have to elaborate a suitable filter that would realize a good compromise.

\section{CONCLUSION}

The concept of ultrasound image-based comanipulation for increasing the feeling of distal contact between an ultrasound probe and an organ despite disturbing friction was introduced in this paper. A first prototype was developed and preliminary experiments have been conducted. The experiments illustrate the potential benefit that the approach could bring to improve the surgeon haptic perception.

These preliminary results are encouraging in that sense that even very basic image processing and control law allowed conclusive improvement brought by the concept. However, we are far yet from proving the feasibility of the approach in a clinical context. Current work consists of building an apparatus that will have enough degrees of freedom (see Sect. III) and an appropriate ultrasonic image processing, in order to perform the tasks on phantom prostate and anatomical models. The images obtained with such phantoms are much more realistic than the "balloon in the water" apparatus. It is expected that the development of a more sophisticated image processing algorithm will be required. Moreover, different control laws will be tested and evaluated.

\section{ACKNOWLEDGMENT}

We would like to thank Michael Baumann and Koelis who provided important information about the Sonix SP and the UroStation. This work is partially supported by ANR TECSAN 2011 program, Prosbot project.

\section{REFERENCES}

[1] A.D. Pearle, D. Kendoff, V. Stueber, V. Musahl and J.A. Repicci, "Perioperative Management of Unicompartmental Knee Arthroplasty Using the MAKO Robotic Arm System MAKoplasty”, in The American journal of orthopedics, vol. 38, pp 16-19, 2009.

[2] W.T. Ang, "Active Tremor Compensation in Handeld Instrument for Microsurgery", PhD thesis from Carnegie Mellon University, 2004.

[3] C.N. Riviere, J. Gangloff and M. de Mathelin, "Robotic Compensation of Biological Motion to Enhance Surgical Accuracy", in Proceeding of the IEEE, vol. 94, No. 9, pp 1705-1716, September 2006.

[4] A. Hassan Zahraee, J. Paik, J. Szewczyk, and G. Morel, "Towards the Development of a Hand-Held Surgical Robot for Laparoscopy", in IEEE/ASME Transactions on Mechatronics, No. 99, pp 1-9, 2010.

[5] H. Kazerooni, "Extender: a case study for human-robot interaction via transfer of power and information signals", IEEE International Workshop and Human Communication, pp 10-20, 1993.

[6] K. Kosuge, Y. Fujisawa, T. Fukuda, "Control of Robot Directly Maneuvered by Operator", in Proceedings of IEEE International Conference on Intelligent Robots and Systems, vol. 1, pp 49-54, Yokohama, July 1993.

[7] X. Lamy, F. Colledani, F. Geffard, Y. Measson and G. Morel, "Human force amplification with industrial robot : Study of dynamic limitations", IEEE/RSJ International Conference on Intelligent Robots and Systems, pp 2487-2494, Taipei, October 2010.

[8] P.J. Berkelman, L.L. Whitcomb, R.H. Taylor and P. Jensen, "A Miniature Instrument Tip Force Sensor for Robot/Human Cooperative Microsurgical Manipulation with Enhanced Force Feedback", MICCAI, pp 897-906, 2000.

[9] G. Picod, A.C. Jambon, P. Dubois and D. Vinatier, "Measurement of the friction in the sheath of the trocars used in laparoscopy", ITBMRBM, vol. 24, pp 217-222, 2003.

[10] RB. Cagneau, G. Morel, D. Bellot, N. Zemiti and G.A. d'Agostino, "A Passive Force Amplifier", in IEEE International Conference on Robotics and Automation, pp 2079-2084, Pasadena, May 2008.

[11] N. Zemiti, G. Morel, T. Ortmaier and N. Bonnet, "Mechatronic Design of a New Robot for Force Control in Minimally Invasive Surgery", IEEE/ASME Transactions on Mechatronics, vol. 12, pp 143-153, April 2007.

[12] C.W. Kennedy and J.P. Desay, "Force Feedback Using Vision", in IEEE International Conference on Advanced Robotics, pp 179-184, Coimbra, 2003.

[13] J. Kim, Y. Kim and J. Kim, "Real-time haptic rendering of slowly deformable bodies based on two dimensional visual information for telemanipulation", in International Conference on Control, Automation and Systems, pp 2448-2451, Seoul, Korea, 2007.

[14] M. Baumann, P. Mozer, V. Daanen, and J. Troccaz, "Transrectal ultrasound prostate biopsy tracking with efficient and accurate deformation estimation", IEEE International Symposium on Biomedical Engineering, pp. 1211-1214, 2009.

[15] M.A. Pastor, B.L. Day, E. Macaluso, K.J. Friston and R.S.J. Frackowiack, "The functional neuroanatomy of temporal discrimination", Journal of Neuroscience, 24(10)2585-2591, March 2004.

[16] A. Jemal, R. Siegel, J. Xu and E. Ward, "Cancer statistics, 2010", CA: A Cancer Jornal for Clinicians, vol. 60, issue 5, pp 277-300, September/October 2010.

[17] A. Villers, D. Mouton, X. Rébillard, D. Chautard and A. Ruffion, "Conditions de réalisation et schéma de ponctions lors d'une première série de biopsies prostatiques", Progrès en Urologie, vol. 14, $\mathrm{n}^{\circ} 2$, pp 144-153, 2004.

[18] M. Marchal, "Modélisation des tissus mous dans leur environnement pour l'aide aux gestes médico-chirurgicaux", PhD thesis from Joseph Fourier University, 2006. 